University of Wollongong

Research Online

Faculty of Engineering and Information

Faculty of Engineering and Information

Sciences - Papers: Part A

Sciences

$1-1-2013$

Transmit antenna subset selection with power balancing for high data rate MIMO-OFDM UWB systems

Ngoc Phuc Le

University of Wollongong, pnl750@uowmail.edu.au

Le Chung Tran

University of Wollongong, Ictran@uow.edu.au

Farzad Safaei

University of Wollongong, farzad@uow.edu.au

Follow this and additional works at: https://ro.uow.edu.au/eispapers

Part of the Engineering Commons, and the Science and Technology Studies Commons

Research Online is the open access institutional repository for the University of Wollongong. For further information contact the UOW Library: research-pubs@uow.edu.au 


\title{
Transmit antenna subset selection with power balancing for high data rate MIMO-OFDM UWB systems
}

\begin{abstract}
This paper proposes per-subcarrier transmit antenna subset selection with power balancing for MIMOOFDM UWB systems to simultaneously improve the system error performance and increase data rates. The deployment of the per-subcarrier antenna subset selection may result in a power unbalance across antennas, which could cause power amplifiers (PAs) to operate in their non-linear regions. To overcome this disadvantage, we formulate a linear optimization problem for the optimal allocation of data subcarriers under a constraint that all antennas have the same number of assigned data symbols. This optimization problem could be applied to systems with an arbitrary number of multiplexed data streams, antennas, and with different selection criteria. The efficacy of the proposed allocation scheme from the PA linearity perspective is validated by analyzing the distribution of the peak amplitude of timedomain signals. Simulation results demonstrate that the proposed system outperforms the system without a balancing constraint.
\end{abstract}

\section{Keywords}

uwb, ofdm, mimo, rate, data, systems, high, transmit, balancing, power, selection, subset, antenna

\section{Disciplines}

Engineering | Science and Technology Studies

\section{Publication Details}

N. Phuc. Le, L. Chung. Tran \& F. Safaei, "Transmit antenna subset selection with power balancing for high data rate MIMO-OFDM UWB systems," in IEEE International Conference on Ultra-Wideband (ICUWB), 2013, pp. 159-164. 


\title{
Transmit Antenna Subset Selection with Power Balancing for High Data Rate MIMO-OFDM UWB Systems
}

\author{
Ngoc Phuc Le, Le Chung Tran, and Farzad Safaei \\ School of Electrical, Computer and Telecommunications Engineering \\ The University of Wollongong \\ Northfields Avenue, NSW 2522, Australia \\ Emails: \{pnl750, lctran, farzad\}@uow.edu.au
}

\begin{abstract}
This paper proposes per-subcarrier transmit antenna subset selection with power balancing for MIMO-OFDM UWB systems to simultaneously improve the system error performance and increase data rates. The deployment of the per-subcarrier antenna subset selection may result in a power unbalance across antennas, which could cause power amplifiers (PAs) to operate in their non-linear regions. To overcome this disadvantage, we formulate a linear optimization problem for the optimal allocation of data subcarriers under a constraint that all antennas have the same number of assigned data symbols. This optimization problem could be applied to systems with an arbitrary number of multiplexed data streams, antennas, and with different selection criteria. The efficacy of the proposed allocation scheme from the PA linearity perspective is validated by analyzing the distribution of the peak amplitude of timedomain signals. Simulation results demonstrate that the proposed system outperforms the system without a balancing constraint.
\end{abstract}

Keywords- MIMO; OFDM-UWB; antenna subset selection; power balancing; linear optimization.

\section{INTRODUCTION}

Ultra-wideband (UWB) has been expected as a technology for delivering gigabit wireless. However, current OFDMUWB (orthogonal frequency division multiplexing-UWB) systems suffer from issues of low data rates and very short transmission range [1]. One of the promising solutions to these issues is MIMO (multi-input multi-output) techniques [1]-[2]. Among various MIMO schemes, antenna selection appears to be promising for OFDM-UWB systems. This is mainly due to a low-cost implementation required for antenna selection [3], and the practicality of this technique in the context of UWB in terms of application scenarios (i.e. indoor operation) as well as equivalent isotropic radiated power (EIRP) restrictions [4].

Some research works have considered the application of antenna selection (AS) to OFDM-UWB systems, e.g. in [5][8]. In these works, selecting antenna on each subcarrier basis, referred to as per-subcarrier selection, was applied to exploit the frequency-selective nature of the UWB channel. Also, many implementation aspects were investigated, including AS with phase precoding for WiMedia compatibility [5], spacefrequency AS with mismatch calibration [6]-[7], or AS with reduced feedback [8]. However, all of these proposed AS schemes were developed for the purpose of performance improvement only. To the best of our knowledge, in all the existing AS-based OFDM-UWB systems in the literature, data are transmitted from only one antenna on each subcarrier. Thus, the achieved spectral efficiency is limited. To fulfill the expectation of delivering gigabit speeds, per-subcarrier antennas subset selection, where multiple data symbols are transmitted simultaneously from multiple antennas on each subcarrier, should be considered for OFDM-UWB systems.

Intuitively, the deployment of per-subcarrier antenna subset selection in OFDM-UWB systems could simultaneously increase data rates resulting from multiplexed data streams and enhance performance by exploiting spatial diversity as well as the frequency-selective of the channel. Besides its advantages, this method has a disadvantage that a large number of data symbols may be assigned on some particular antennas. The power amplifiers (PAs) associated with those antennas may operate in the nonlinear region due to a large power, which degrades system performance. While the design of PAs with a larger dynamic range could alleviate this issue, it is so demanding for low-cost UWB devices. Another approach is selecting antenna subsets with a constraint that the number of data subcarriers allocated to each antenna is equal. Also, the constrained scheme should result in a minimal loss of performance compared to an unconstrained scheme. In [9], the authors have considered linear optimization to devise such a scheme. However, the formulated problem in [9] is only applicable to AS schemes where one antenna is active on each subcarrier, e.g. [5]-[8]. Moreover, even though the rationale of this method comes from the perspective of PAs, the analysis of its benefits from a PAs viewpoint has not been addressed.

In this paper, we propose per-subcarrier antenna subset selection with power balancing for MIMO-OFDM UWB systems. The major contributions of this work are as follows.

- A linear optimization problem is formulated to achieve an optimal solution for the constrained selection in the systems with an arbitrary number of multiplexed data streams.

- A reduced-complexity strategy that requires small feedback bits and lower effort to solve the optimization is proposed by exploiting the correlation between adjacent subcarriers.

- The effectiveness of the proposed per-subcarrier antenna subset selection with power balancing is analyzed from a PAs perspective by deriving the CCDF (complementary cumulative distribution function) of the peak amplitude of per-subcarrier antenna selection MIMO-OFDM signals.

Numerical results are also provided to verify the analyses and demonstrate the improvement in terms of error performance. 


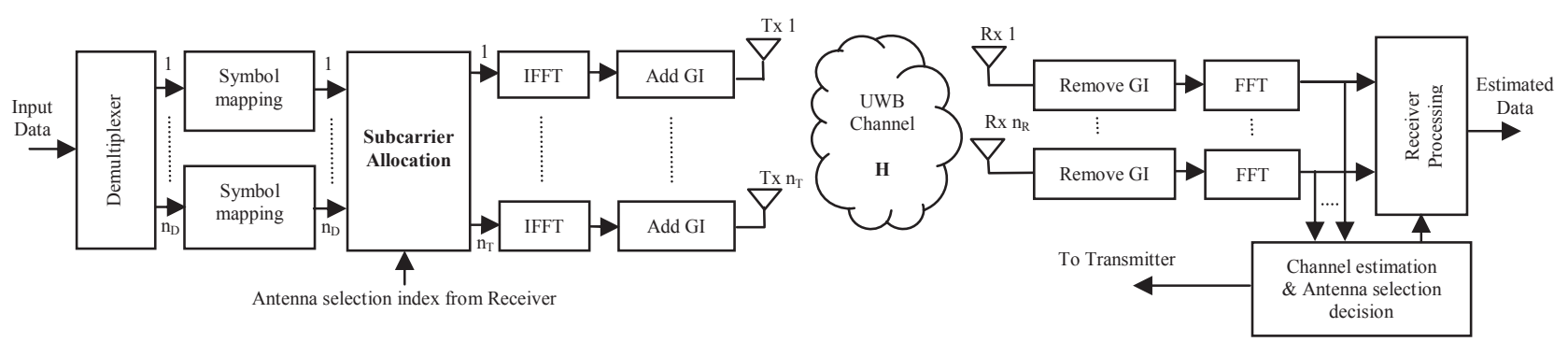

Fig. 1 A simplified block diagram of a MIMO-OFDM UWB system with transmit antenna subset selection

Notation: A bold letter denotes a vector or a matrix, whereas an italic letter denotes a variable. $(.)^{*},(.)^{\mathrm{T}},(.)^{H},(.)^{-1}, \otimes, E\{$.$\} ,$ and $\operatorname{det}($.$) denote complex conjugation, transpose, Hermitian$ transpose, inverse, the Kronecker product, expectation, and determinant of a matrix, respectively. $\mathbf{I}_{n}$ indicates the $n \times n$ identity matrix, and $\mathbf{1}_{K}$ is a $K \times 1$ vector of ones. $\mathfrak{R}$ indicates the set of real numbers.

\section{PER-SUBCARRIER ANTENNA SUBSET SELECTION FOR MIMO-OFDM UWB SYSTEMS}

\section{A. System Model}

We consider a MIMO-OFDM UWB system with $K$ subcarriers, $n_{T}$ transmit antennas, and $n_{R}$ receive antennas as shown in Fig.1. At the transmitter, the input data are demultiplexed into $n_{D}$ independent streams, where $n_{D}<n_{T}$ and $n_{D} \leq n_{R}$. Each data stream is then mapped onto $M$-QAM (Quadrature Amplitude Modulation) constellations. Denote $u_{l}^{k}, 1 \leq l \leq n_{D}, 0 \leq k \leq K-1$, and $x_{i}^{k}, 1 \leq i \leq n_{T}$, to be the symbols that the subcarrier block takes at its $l^{\text {th }}$ input and outputs at its $i^{\text {th }}$ output, respectively. The allocation block assigns the elements of $\mathbf{u}_{k}=\left[u_{1}^{k}, u_{2}^{k}, \ldots, u_{n_{D}}^{k}\right]^{T}$ to $n_{D}$ selected antennas at the $k^{\text {th }}, 0 \leq k \leq K-1$, subcarrier based on feedback information. As a result, only $n_{D}$ elements in a vector $\mathbf{x}_{k}=\left[x_{1}^{k}, x_{2}^{k}, \ldots, x_{n_{T}}^{k}\right]^{T}$ are assigned values from $\mathbf{u}_{k}$, whereas the others are zeros. Here, we assume that $E\left\{\mathbf{u}_{k} \mathbf{u}_{k}^{H}\right\}=\sigma^{2} \mathbf{I}_{n_{D}}$. The output sequences from the subcarrier allocation block are then fed into $K$-point IFFT blocks. The discrete-time baseband OFDM signals can be expressed as ${ }^{1}$

$$
s_{i}(n)=\frac{1}{\sqrt{K}} \sum_{k=0}^{K-1} x_{i}^{k} e^{j 2 \pi n k / K}, 0 \leq n \leq K-1,1 \leq i \leq n_{T} .
$$

Each time-domain OFDM signal is then added with a guard interval (GI) before being transmitted via its corresponding transmit antenna. At the receiver, the received signal at each antenna is fed into the FFT block after the GI is removed. The system model in the frequency domain corresponding to the $k^{\text {th }}$ subcarrier can be expressed as

$$
\begin{aligned}
\mathbf{y}_{k} & =\mathbf{H}_{k} \mathbf{x}_{k}+\mathbf{n}_{k} \\
& =\underline{\mathbf{H}}_{k} \mathbf{u}_{k}+\mathbf{n}_{k},
\end{aligned}
$$

${ }^{1}$ Without loss of generality, we consider the Nyquist sampling signal in this paper. where

$$
\begin{aligned}
\mathbf{x}_{k}= & {\left[\begin{array}{llll}
x_{1}^{k} & x_{2}^{k} & \ldots & x_{n_{T}}^{k}
\end{array}\right]^{T}, } \\
\mathbf{H}_{k} & =\left[\begin{array}{cccc}
h_{1,1}^{k} & h_{1,2}^{k} & \ldots & h_{1, n_{T}}^{k} \\
h_{2,1}^{k} & h_{2,2}^{k} & \ldots & h_{2, n_{T}}^{k} \\
\ldots & \ldots & \ldots & \ldots \\
h_{n_{R}}^{k}, 1 & h_{n_{R}, 2}^{k} & \ldots & h_{n_{R}, n_{T}}^{k}
\end{array}\right], \\
\mathbf{y}_{k} & =\left[\begin{array}{llll}
y_{1}^{k} & y_{2}^{k} & \ldots & y_{n_{R}}^{k}
\end{array}\right]^{T}, \\
\mathbf{n}_{k} & =\left[\begin{array}{llll}
n_{1}^{k} & n_{2}^{k} & \ldots & n_{n_{R}}^{k}
\end{array}\right]^{T} .
\end{aligned}
$$

In the above equations, $h_{j, i}^{k}$ indicates the channel coefficient from the $i^{\text {th }}$ transmit antenna to the $j^{\text {th }}$ receive antenna. The effective channel matrix $\underline{\mathbf{H}}_{k}$ is obtained by eliminating the columns of $\mathbf{H}_{k}$ corresponding to the unselected transmit antennas. Also, $y_{j}^{k}$ and $n_{j}^{k}$ denote the received signal and the noise at the $j^{\text {th }}$ receive antenna, respectively. Here, the noise is modeled as a Gaussian random variable with zero mean and $E\left\{\mathbf{n}_{k} \mathbf{n}_{k}^{H}\right\}=\sigma_{n}^{2} \mathbf{I}_{n_{R}}$. We assume that per-subcarrier power loading is not an option due to the strict regulation of a power spectral mask in UWB systems. Finally, various MIMO detection techniques can be used to detect signals. For simplicity, we only consider a ZF (zero-forcing) receiver.

\section{B. Per-subcarrier Antenna Subset Selection}

In a MIMO-OFDM UWB system with per-subcarrier subset selection, antenna subsets are selected independently for each subcarrier. On each subcarrier, only $n_{D}$ antennas out of $n_{T}$ available transmit antennas are active. Denote $\boldsymbol{\Gamma}_{\gamma}, \gamma=1,2, \ldots, \Gamma$ to be the $\gamma^{\text {th }}$ subset consisting of $n_{D}$ selected antennas, where $\Gamma=\left(\begin{array}{c}n_{D} \\ n_{T}\end{array}\right)=\frac{n_{T} !}{n_{D} !\left(n_{T}-n_{D}\right) !}$ is the number of all possible $n_{D}$-element subsets. Each subset consists of $n_{D}$ transmit antenna indices that are chosen based on the feedback information from the receiver. For example, when $n_{T}=4$ and $n_{D}=2$, then $\Gamma=6$, and all possible subsets $\Gamma_{\gamma}, \gamma=1,2, \ldots, 6$ are defined in the Table I. The choice of the best antenna subset depends on a particular selection criterion.

Several antenna selection criteria, such as maximum capacity [10], maximum SNR (signal-to-noise ratio) [10], or MMSE (minimum mean-squared error) [11], can be extended 
TABLE I. ANTENNA SUBSETS

$\left(n_{T}=4, n_{D}=2\right.$, and $\left.\Gamma=6\right)$

\begin{tabular}{|c|c|}
\hline$\gamma$ & $\Gamma_{\gamma}$ \\
\hline 1 & $\{1,2\}$ \\
\hline 2 & $\{1,3\}$ \\
\hline 3 & $\{1,4\}$ \\
\hline 4 & $\{2,3\}$ \\
\hline 5 & $\{2,4\}$ \\
\hline 6 & $\{3,4\}$ \\
\hline
\end{tabular}

to this system. For the sake of brevity, we only investigate a MMSE criterion in this paper. This criterion selects the best antenna subset from the viewpoint of minimum mean-squared error. In other words, it aims to minimize the error rate. When a $\mathrm{ZF}$ receiver is used, the mean-squared error (MSE) between the estimated data and the transmitted data corresponding to the $k^{\text {th }}$ subcarrier and subset $\Gamma_{\gamma}$ is given by [11]

$$
\mathbf{M S E}_{\gamma}^{k}=\sigma_{n}^{2}\left(\underline{\mathbf{H}}_{k}^{H} \underline{\mathbf{H}}_{k}\right)^{-1} \text {. }
$$

As this system considers the per-subcarrier selection rather than a bulk selection (i.e. choosing the same subset for all subcarriers) as in [11], a formulated problem is different from the one in [11]. Specifically, the subset at the $k^{\text {th }}$ subcarrier is determined by minimizing the trace of the MSE matrix, i.e.

$$
\Gamma_{\gamma}(k)=\arg \min _{\gamma=1, \ldots, \Gamma} \operatorname{trace}\left\{\mathbf{M S E}_{\gamma}^{k}\right\} .
$$

With respect to a feedback mechanism used in this system, the selected antenna indexes could be directly transmitted through reverse links in a TDD (time-division duplex) mode. Also, in UWB indoor scenarios, the channel might not be changed during the transmission of several consecutive frames. Hence, the transmitter will reallocate data subcarriers according to the updated feedback information. In MIMOOFDM systems with large values of $\Gamma$ and/or $K$, the number of feedback bits might be high. Reduced feedback could be realized by combining subcarriers into a cluster and using only one antenna subset for all subcarriers in the cluster [8]. Thus, with the cluster size of $L$, the number of feedback bits is reduced by $1 / L$. We propose the following criterion for choosing the subset for the $m^{\text {th }}$ cluster, $1 \leq m \leq M, M=K / L$,

$$
\boldsymbol{\Gamma}_{\gamma}(m)=\arg \min _{\gamma=1, \ldots, \Gamma}\left\{\sum_{k=(m-1) L+1}^{m L} \operatorname{trace}\left\{\mathbf{M S E}_{\gamma}^{k}\right\}\right\} .
$$

\section{OPTIMIZATION FOR SUBCARRIER ALLOCATION WITH POWER BALANCING}

In Section II, we have described the MIMO-OFDM UWB system with per-subcarrier transmit antenna subset selection. In general, during each OFDM symbol duration, the number of data subcarriers assigned to each transmit antenna might be significantly different depending on the channel condition. This will lead to performance degradation as power amplifiers may operate in their nonlinear regions. To deal with this problem, one possible approach is selecting antennas under a constraint that all antennas have the same number of data subcarriers as illustrated in Fig. 2. Moreover, the constrained selection scheme should result in a minimum loss of capacity or error performance compared to an unconstrained scheme. To this end, we formulate a linear optimization problem for

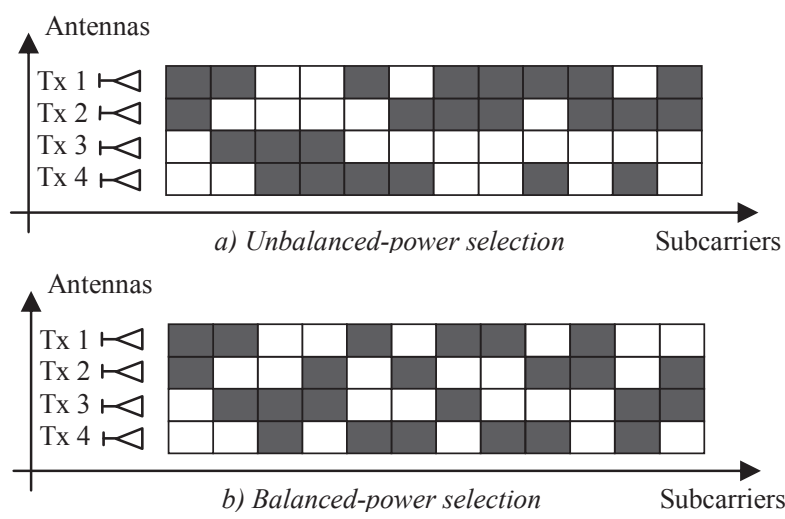

Fig. 2 Illustration of per-subcarrier antenna subset selection $\left(n_{T}=4, n_{D}=2\right.$, and $\left.K=12\right)$.

the optimal allocation of data subcarriers in MIMO-OFDM UWB systems with arbitrary numbers of multiplexed data streams $n_{D}$ and transmit antennas $n_{T}\left(n_{D}<n_{T}\right)$.

We define a variable $z_{\gamma}^{k}$, where $z_{\gamma}^{k}=1$ if $\boldsymbol{\Gamma}_{\gamma}$ is chosen for the $k^{\text {th }}$ subcarrier, and $z_{\gamma}^{k}=0$ otherwise. Also, denote $c_{\gamma}^{k}$ to be the cost associated with the chosen subset $\boldsymbol{\Gamma}_{\gamma}$. The type of the cost depends on antenna selection criteria, e.g. $c_{\gamma}^{k}=\operatorname{trace}\left\{\mathbf{M S E}_{\gamma}^{k}\right\}$ if the MMSE selection criterion is used. The total cost function can be expressed as

$$
f=\sum_{k=0}^{K-1} \sum_{\gamma=1}^{\Gamma} c_{\gamma}^{k} z_{\gamma}^{k}
$$

As mentioned earlier, in this system, only $n_{D}$ antennas transmit data symbols on each subcarrier. This is equivalent to choosing only one subset of $n_{D}$ elements $\Gamma_{\gamma}, \gamma=1,2, \ldots, \Gamma$ per subcarrier. Thus, the first constraint can be expressed as

$$
\sum_{\gamma=1}^{\Gamma} z_{\gamma}^{k}=1, \forall k=0,1, \ldots, K-1 .
$$

The second constraint is that all transmit antennas have the same number of allocated data subcarriers. Note that, in the case of $K n_{D}$ is not divisible by $n_{T}$, some antennas will allowed to have one more subcarrier than others. This will guarantee that the transmit power will be evenly distributed over the transmit antennas as much as it could. This constraint can be expressed as

$$
\sum_{k=0}^{K-1} z_{\gamma}^{k}=\lambda_{\gamma}, \gamma=1,2, \ldots, \Gamma,
$$

where the parameter $\lambda_{\gamma}$ is the number of times that the subset $\boldsymbol{\Gamma}_{\gamma}$ is chosen, and its value depends on the specific values of $K, n_{D}$, and $n_{T}$. In case of $K$ is divisible by $\Gamma$, the expression for calculating $\lambda_{\gamma}$ can be given by

$$
\lambda_{\gamma}=\frac{K}{\Gamma}, \forall \gamma=1,2, \ldots, \Gamma \text {. }
$$

For example, if $n_{T}=4, n_{D}=2$, and $K=12$, then $\lambda_{\gamma}=\frac{12}{6}=2$, $\forall \gamma=1, \ldots, 6$. As all the subsets are chosen twice, from Table I, 
we know that all transmit antennas will have six data symbols (cf. Fig. 2b).

The optimization problem in the proposed system is now a minimization of the cost function (10) subject to two constraints (11) and (12). Note that, in the system without power balancing, a subcarrier allocation problem is equivalent to minimizing (10) subject to the constraint (11) only.

In the following, we will represent the above optimization problem in a matrix form. Let us define a vector $\mathbf{z}=\left(z_{1}^{0}, \ldots, z_{\Gamma}^{0}, z_{1}^{1}, \ldots, z_{\Gamma}^{1}, \ldots, z_{1}^{K-1}, \ldots, z_{\Gamma}^{K-1}\right)^{T} \in\{0,1\}^{K \Gamma \times 1}, \quad$ and $\mathrm{a}$ cost vector $\mathbf{c}=\left(c_{1}^{0}, \ldots, c_{\Gamma}^{0}, c_{1}^{1}, \ldots, c_{\Gamma}^{1}, \ldots, c_{1}^{K-1}, \ldots, c_{\Gamma}^{K-1}\right)^{T} \in \mathfrak{R}^{K \Gamma \times 1}$. Then, (10) can be rewritten as $f=\mathbf{c}^{T} \mathbf{z}$. Also, the first and the second constraints can now be expressed as

$$
\mathbf{A}_{1} \mathbf{z}=\mathbf{1}_{K},
$$

where $\mathbf{A}_{1}=\mathbf{I}_{K} \otimes \mathbf{1}_{\Gamma}^{T} \in\{0,1\}^{K \times K \Gamma}$, and

$$
\mathbf{A}_{2} \mathbf{z}=\boldsymbol{\lambda},
$$

where $\quad \mathbf{A}_{2}=\mathbf{1}_{K}^{T} \otimes \mathbf{I}_{\Gamma} \in\{0,1\}^{\Gamma \times K \Gamma} \quad$ and $\quad \lambda=\left(\lambda_{1}, \lambda_{2}, \ldots, \lambda_{\Gamma}\right)^{T}$. Moreover, these constraints could be combined in a concise form as

$$
\mathbf{A z}=\mathbf{a},
$$

where $\mathbf{A}=\left(\mathbf{A}_{1}^{T}, \mathbf{A}_{2}^{T}\right)^{T} \in\{0,1\}^{(K+\Gamma) \times K \Gamma}$ and $\mathbf{a}=\left(\mathbf{1}_{K}^{T}, \lambda^{T}\right)^{T}$. Consequently, the optimization problem becomes

$$
\begin{gathered}
\min _{z \in\{0,1\}^{K \Gamma \times 1}} \mathbf{c}^{T} \mathbf{z}, \\
\text { s.t. } \quad \mathbf{A z}=\mathbf{a} .
\end{gathered}
$$

It is obvious that (17) has a canonical form of a binary linear optimization problem. Moreover, it can be shown that the constraint matrix $\mathbf{A}$ is a totally unimodular matrix ${ }^{2}$. Thus, this binary linear optimization problem can be relaxed to linear programming [12]. As a result, the optimization problem in (17) can be solved efficiently by well-known linear programming methods, such as simplex methods or interior point method [13].

In the proposed system with reduced feedback, the optimization problem is formulated on a cluster basis rather than on a subcarrier basis. More specifically, the optimization is similar to (17), excepting that: i) the number of variable is $\Gamma K / L$, i.e., $\mathbf{z} \in\{0,1\}^{(K \Gamma / L) \times 1}$; ii) a cost vector $\mathbf{c} \in \Re^{(K \Gamma / L) \times 1}$ and its elements are now $c_{\gamma}^{m}=\sum_{k=(m-1) L+1}^{m L} \operatorname{trace}\left\{\mathbf{M S E} \mathbf{E}_{\gamma}^{k}\right\}$; iii) matrix $\mathbf{A}$ and vector $\mathbf{a}$ in the constraint will need to be changed accordingly. It is well-known that the complexity to solve linear optimization is polynomial in the number of variables and the bit size of the problem [13]. In other words, the complexity depends on the values of $\Gamma$ and $K$. Therefore, performing the optimization in this case will require much lower computational effort compared to that on a subcarrier basis (i.e. (17)). As a result, with this combined strategy, the proposed system could enjoy both small feedback overhead and low complexity for optimization.

\footnotetext{
${ }^{2}$ Proof is similar to that in [9, Appendix A].
}

\section{Statistical Distribution of PEAK AMPlitudes}

In this section, we analyze the efficacy of the antenna subset selection MIMO-OFDM UWB system with power balancing over its counterpart. As mentioned earlier, when there is no the power balancing constraint, the number of data symbols (i.e. data subcarriers) allocated on each antenna might be significantly different depending only on the channel condition. On the other hand, it is highly likely that a larger number of allocated data subcarriers will lead to a higher peak power of a time-domain signal. As a result, the peak power on each antenna might vary significantly between OFDM symbol periods as well as among antennas. This will definitely affects the efficiency of the PAs, which in turn reduces the potential benefits of the system [14]. Therefore, it is important to investigate the statistical distribution of the peak amplitude (or the peak power) of the antenna selection MIMO-OFDM signals. To this end, we derive the complementary cumulative distribution function $(C C D F)$ of the peak amplitude $A$ of the per-subcarrier antenna selection MIMO-OFDM signals. This $C C D F$ is defined as the probability that the peak amplitude $A$ of the OFDM signals exceeds a given threshold $A_{0}$, i.e.

$$
\operatorname{CCDF}\left(A_{0}\right)=\operatorname{Pr}\left(A>A_{0}\right) \text {. }
$$

Let us begin with the discrete-time OFDM signal $s_{i}(n), n=0,1, \ldots, K-1$, corresponding to the $i^{\text {th }}$ transmit antenna. The peak amplitude of this signal is defined as

$$
A_{i}=\max _{0 \leq n \leq K-1}\left|s_{i}(n)\right| .
$$

For analytical tractability, we assume that both the real part and imaginary part of the signal $s_{i}(n)$ are asymptotically independent and identically distributed Gaussian random variables. Note that this assumption, which is based on the central limit theorem [15], only holds when the number of assigned data subcarriers on the $i^{\text {th }}$ antenna, denoted as $K_{i}$, is large enough ${ }^{3}$. As a result, $\left|s_{i}(n)\right|$ follows the Rayleigh distribution with the probability density function [15]

$$
p_{|s|}\left(\left|s_{i}\right|\right)=\frac{2\left|s_{i}\right|}{\sigma_{K_{i}}^{2}} e^{-\left|s_{i}\right|^{2} / \sigma_{K_{i}}^{2}},
$$

where $\sigma_{K_{i}}^{2}=\sigma^{2} K_{i} / K$ is the variance of the signal $\left|s_{i}(n)\right|$. Note that $\sum_{i=1}^{n_{T}} K_{i}=n_{D} K$, thus we have $\sum_{i=1}^{n_{T}} \sigma_{K_{i}}^{2}=n_{D} \sigma^{2}$. The $C D F$ (cumulative distribution function) of the signal $\left|s_{i}(n)\right|$ is given as

$$
\operatorname{Pr}\left(\left|s_{i}\right| \leq \rho\right)=1-e^{-\rho^{2} / \sigma_{K_{i}}^{2}}, \rho \geq 0 .
$$

Suppose that $K$ samples of $\left|s_{i}(n)\right|, n=0,1, \ldots, K-1$, are independent, the $C D F$ of the peak amplitude $A_{i}$ can be expressed as

$$
\begin{aligned}
C D F_{A_{i}} & =\operatorname{Pr}\left(A_{i} \leq A_{0}\right) \\
& =\operatorname{Pr}\left(\left|s_{i}(0)\right| \leq A_{0}\right) \operatorname{Pr}\left(\left|s_{i}(1)\right| \leq A_{0}\right) \ldots \operatorname{Pr}\left(\left|s_{i}(K-1)\right| \leq A_{0}\right) \\
& =\left(1-e^{-A_{0}^{2} / \sigma_{K_{i}}^{2}}\right)^{K}
\end{aligned}
$$

\footnotetext{
${ }^{3}$ In Fig. 2a, we have $K_{1}=8, K_{2}=7, K_{3}=3$ and $K_{4}=6$.
} 
TABle II. Simulation Parameters [16].

\begin{tabular}{|l|l|}
\hline \multicolumn{1}{|c|}{ Parameter } & \multicolumn{1}{|c|}{ Value } \\
\hline Sampling frequency & $528 \mathrm{MHz}$ \\
FFT size & 128 \\
Number of samples in zero-padded suffix & 37 \\
Modulation scheme & MDCM (Modified Dual \\
& Carrier Modulation) \\
LDPC code (Table 6.31 in [16]) & Code rate: 3/4; 10 iterations \\
IEEE 802.15.3a channel model & CM1 \\
\hline
\end{tabular}

In a MIMO-OFDM UWB system with nonlinear power amplifiers, the peak amplitudes of signals on all transmit antennas should be simultaneously as small as possible. The peak amplitude $A$ of the system could be defined as

$$
A=\max _{1 \leq i \leq n_{T}} A_{i} .
$$

Given the statistical independence of data among transmit antennas, which is the case in the considered spatial multiplexed OFDM system, the $C D F$ of the peak amplitude $A$ is calculated as

$$
\begin{aligned}
C D F_{A} & =\operatorname{Pr}\left(A \leq A_{0}\right)=\operatorname{Pr}\left(A_{1} \leq A_{0}\right) \operatorname{Pr}\left(A_{2} \leq A_{0}\right) \ldots \operatorname{Pr}\left(A_{n_{T}} \leq A_{0}\right) \\
& =\prod_{i=1}^{n_{T}}\left(1-e^{-A_{0}^{2} / \sigma_{K_{i}}^{2}}\right)^{K} .
\end{aligned}
$$

Therefore, the $C C D F$ of the peak amplitude of the antenna selection MIMO-OFDM signals can be expressed as

$$
C C D F_{\text {unbalanced }}\left(A_{0}\right)=1-C D F_{A}=1-\prod_{i=1}^{n_{T}}\left(1-e^{-A_{0}^{2} / \sigma_{K_{i}}^{2}}\right)^{K} \text {. }
$$

For the case of the MIMO-OFDM UWB system with a power balancing constraint, the total number of allocated data subcarriers per transmit antenna is equal to one another. Thus, we have $\sigma_{K_{i}}^{2}=n_{D} \sigma^{2} / n_{T} \ddot{=} \sigma_{\bar{K}}^{2}, \forall i=1,2, \ldots, n_{T}$. As a result, the $C C D F$ expression could be simplified to as

$$
C C D F_{\text {balanced }}\left(A_{0}\right)=1-\left(1-e^{-A_{0}^{2} / \sigma_{\bar{K}}^{2}}\right)^{n_{T} K} \text {. }
$$

It can be shown that the value $C C D F_{\text {balanced }}\left(A_{0}\right)$ is smaller than $C C D F_{\text {unbalanced }}\left(A_{0}\right)$. Therefore, from the PAs perspective, the proposed system with power balancing is better than its counterpart. In addition, a large power back-off is required in the system without power balancing constraint to avoid error floor. As a result, performance degradation is inevitable in this system. Performance comparison based on numerical results will be provided and discussed in the next section.

\section{Simulation Results}

We consider a MIMO-OFDM UWB system with $n_{T}=4$ and $n_{D}=2$ in our simulations. The simulation parameters are listed in Table II. These parameters are chosen based on the legacy WiMedia MB-OFDM UWB (Multiband-OFDM UWB) standard [16] for a data rate of $960 \mathrm{Mbps}$. Therefore, the data rate in the proposed system when $n_{D}=2$ is 1920 Mbps. We measure the system performance in terms of packet-error rate (PER) over the channel models of CM1 defined in the IEEE 802.15.3a channel model [17]. The channel CM1 is based on a measurement of a line-of-sight scenario where the distance between the transmitter and the receiver (Tx-Rx) is up to $4 \mathrm{~m}$. Moreover, the multipath gains are modeled as independent

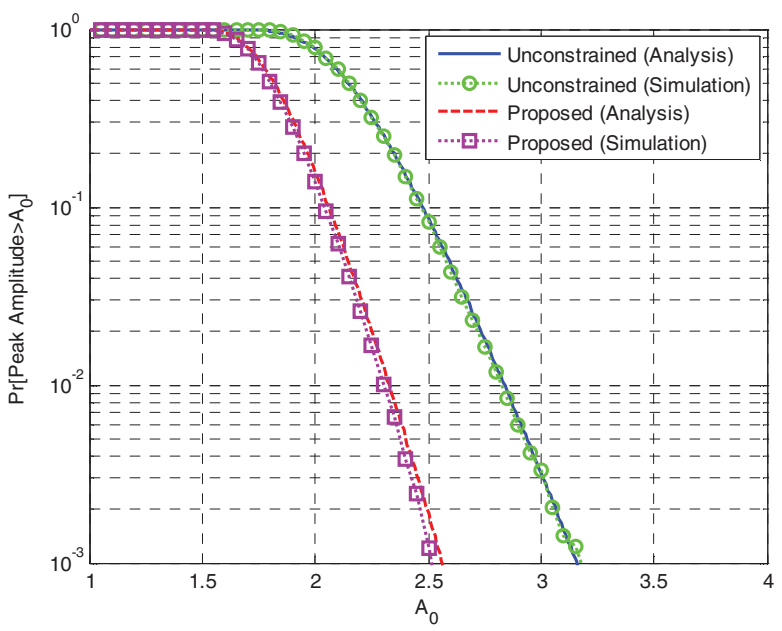

Fig. 3 Comparison of the $C C D F$ s of the peak amplitudes of antenna selection MIMO-OFDM signals.

log-normally distributed random variables. We assume that perfect channel state information is available at the receiver. Also, the feedback link has no delay and is error-free.

In Fig. 3, we plot the CCDFs of the peak amplitude of antenna selection MIMO-OFDM signals. Both theoretical and simulation results are presented in the figure. Here, subcarrier allocation patterns are obtained by running simulations with a $\mathrm{ZF}$ receiver and the MMSE selection criterion at $\mathrm{SNR}=15$ $\mathrm{dB}$. Also, the average energy of transmitted data symbols is normalized to unity, i.e. $\sigma^{2}=1$. The simulation results confirm that the proposed system with power balancing offers a better $C C D F$ than its counterpart. In addition, it is important to note that the analytic curves according to (25) and (26) are close to the simulation curves. The small gaps exist due to the fact that the assumptions in the derivation described in Section IV do not strictly hold. In particular, the assumption of independent samples $\left|s_{\mathrm{i}}(n)\right|$ to obtain (22) is not strictly true as we have $\sum_{n=0}^{K-1}\left|s_{i}(n)\right|^{2}=K_{i}$ by Parseval's relation [15]. Moreover, the number of allocated data symbols on some antennas may be not large enough for (20) to be fully valid.

To demonstrate the superiority of the proposed system over the system without the power-balancing constraint in terms of error performance, we simulate the systems with nonlinear power amplifiers. We consider nonlinear PAs with ideal predistortion (i.e. soft envelope limiter) with input back-off of $8 \mathrm{~dB}$. The power back-off is required on the antennas where the number of assigned data symbols is large to avoid error floor. We remind that power loading is not considered in this paper due to the EIRP restrictions. Also, to obtain proper decision variables for symbol detection, compensation for all attenuations introduced by the nonlinear PAs need to be included [18]. Fig. 4 compares the performance of the two systems. It can be seen that there is a significant improvement in terms of PER performance in the proposed system. Also, a value of $\mathrm{PER}=10^{-2}$ could be achieved at a low SNR region. The performance of the system without power balancing is degraded due to the fact that the large power back-offs will reduce the received signal-to-noise-plus-distortion ratio. 


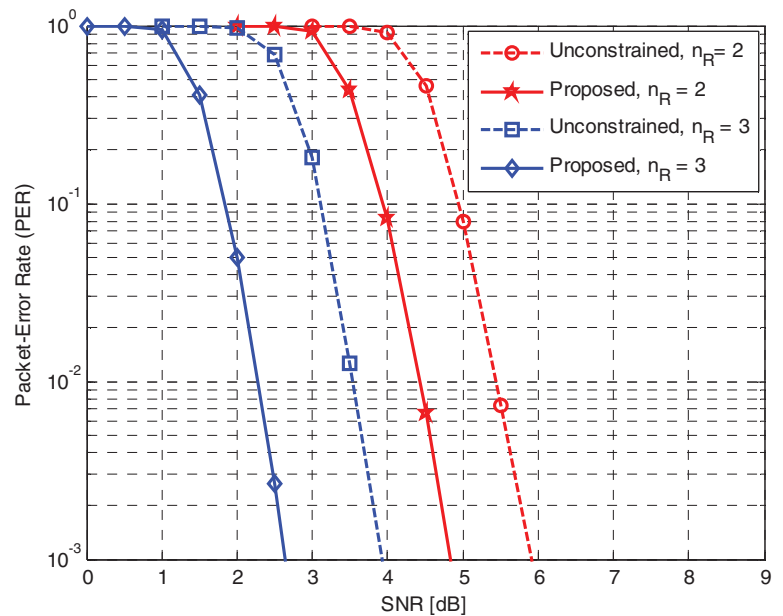

Fig. 4 Comparison of the packet-error rate (PER) performance.

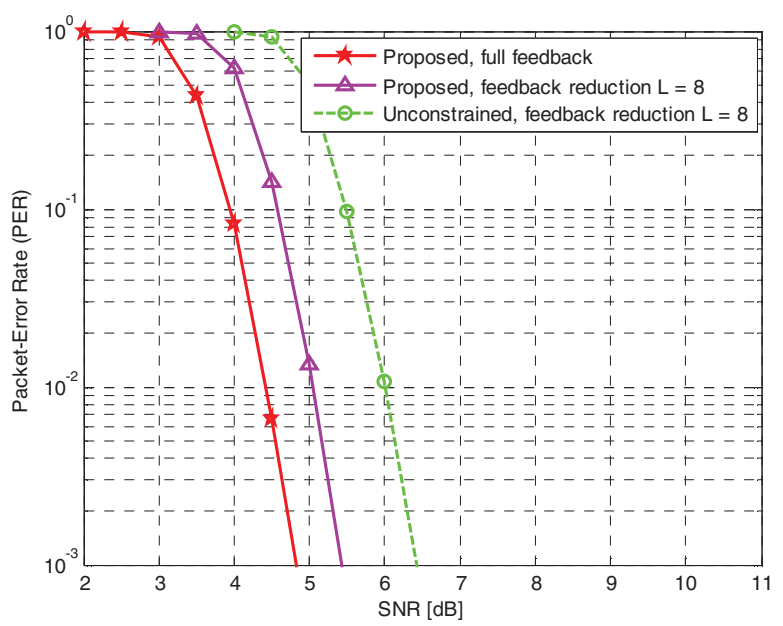

Fig. 5 PER performance of the proposed system with the reducedcomplexity approach $\left(n_{R}=2\right)$.

In Fig. 5, we plot the PER performance of the proposed system with the reduced-complexity approach. Here, the feedback reduction of $L=8$ is used. As predicted, there is some loss in performance when applying feedback reduction compared to full feedback. However, the system with feedback reduction requires only $12.5 \%$ of the number of feedback bits and has lower computational effort for solving the optimization problem. In addition, the proposed system with power balancing still outperforms its counterpart under reduced feedback. These results illustrate the efficiency of the proposed per-subcarrier antenna subset selection MIMOOFDM UWB system with power balancing for the future generations of OFDM based-UWB communications.

\section{CONCLUSIONS}

In this paper, we have proposed per-subcarrier antenna subset selection with power balancing for MIMO-OFDM UWB systems to simultaneously increase data rates and improve system performance (and/or extend transmission range). To deal with the issue of power unbalancing across antennas, we have formulated a linear optimization problem to equally allocate data subcarriers among the transmit antennas.
This formulated optimization can be solved efficiently by existing methods. Moreover, we have developed the reducedcomplexity approach that requires small feedback overhead and lower computational effort for solving the optimization problem. We have derived the CCDF of the peak amplitudes of the time-domain MIMO-OFDM signals and have shown that, from the perspective of PAs, the proposed optimal allocation scheme outperforms the scheme without power balancing. Simulation results have been provided to confirm this benefit. The results have also shown that a significant improvement in terms of error performance could be achieved in the system with power balancing compared to its counterpart when the nonlinear PAs are considered.

\section{REFERENCES}

[1] V. Sipal, B. Allen, D. Edwards, and B. Honary, "Twenty years of ultrawideband: opportunities and challenges," IET Communications, vol. 6, no. 2, pp. 1147-1162, July 2012.

[2] T. Kaiser, F. Zheng, and E. Dimitrov, "An overview of ultrawideband systems with MIMO,” Proc. of the IEEE, vol. 97, no. 2, Feb. 2009.

[3] A. F. Molisch and M. Z. Win, "MIMO systems with antenna selection," IEEE Microwave Magazine, vol. 5, no. 1, pp. 45-56, March 2004.

[4] C. M. Vithanage, J. P. Coon, and S. C. J. Parker, "On capacity-optimal precoding for multiple antenna systems subject to EIRP restrictions," IEEE Trans. Wireless Commun., vol. 7, no. 12, pp. 5182-87, Dec. 2008.

[5] C. M. Vithanage, S. C. J. Parker, and M. Sandell, "Antenna selection with phase precoding for high performance UWB communication with legacy WiMedia multiband OFDM devices," in Proc. IEEE Intern. Conf. Commun., ICC 2008, pp. 3938-3942, May 2008.

[6] X. Peng, Z. Lin, F. Chin, and K. B. Png, "Space-frequency transmit selection for MB-OFDM UWB communication systems with multiple transmit antennas," in Proc. IEEE Int. Conf. Ultra-Wideband, ICUWB 09, pp. 788 -792, Sep. 2009.

[7] Z. Lin, X. Peng, F. Chin, and K. B. Png, "Enhanced MB-OFDM UWB system with multiple transmit and receive antennas," in Proc. IEEE Int. Conf. Ultra-Wideband, ICUWB 09, pp. 793 -797, Sep. 2009.

[8] K. Kim, J. Park, J. Koo, Y. Kim, and J. Kim, "Enhanced WiMedia UWB system using antenna selection with reduced feedback," in Proc. IEEE Consumer Communications and Networking Conference, CCNC 2012, pp. 843-848, Jan. 2012.

[9] M. Sandell and J. Coon, "Per-subcarrier antenna selection with power constraints in OFDM systems", IEEE Transactions on Wireless Communications, vol. 8, no. 2, pp. 673-677, Feb. 2009.

[10] R.W. Heath, A. Paulraj, and S. Sandhu, "Antenna selection for spatial multiplexing systems with linear receivers," IEEE Communications Letters, vol. 5, pp. 142-144, April 2001.

[11] Y. Liu, Y. Zhang, C. Ji, W. Q. Malik, and D. J. Edwards, "A low complexity receive antenna selection algorithm for MIMO-OFDM wireless systems," IEEE Transactions on Vehicular Technology, vol. 58, no. 6, pp. 2793-2802, July 2009.

[12] L. A. Wolsey, Integer Programming, $1^{\text {st }}$ edition, John Willey \& Sons, 1998.

[13] R. Fletcher, Practical Methods of Optimization, $2^{\text {nd }}$ edition, John Willey \& Sons, 1987.

[14] R. Prasad, OFDM for Wireless Communications Systems, $1^{\text {st }}$ edition, Artech House, 2004.

[15] J. G. Proakis, Digital Communications, $4^{\text {th }}$ ed., McGraw Hill Inc., 2001.

[16] A. Batra et al., "Multiband OFDM physical layer specification," WiMedia Alliance, Release 1.5, August 2009.

[17] J. Foerster et al., "Channel modeling sub-committee report final," IEEE P802.15-02/490r1-SG3a, Feb. 2003.

[18] P. Banelli, "Theoretical analysis and performance of OFDM signals in nonlinear fading channels," IEEE Transactions on Wireless Comm., vol. 2, no. 2, pp. 284-293, March 2003. 